\title{
Lattice gas with nearest- and next-to-nearest-neighbor exclusion
}

\author{
Xiaomei Feng, ${ }^{1,2}$ Henk W. J. Blöte, ${ }^{3,2}$ and Bernard Nienhuis ${ }^{4}$ \\ ${ }^{1}$ Nanjing University of Aeronautics and Astronautics, 29 Yudao St., 210016 Nanjing, People's Republic of China \\ ${ }^{2}$ Faculty of Applied Sciences, Delft University of Technology, P. O. Box 5046, 2600 GA Delft, The Netherlands \\ ${ }^{3}$ Instituut Lorentz, Leiden University, P. O. Box 9506, 2300 RA Leiden, The Netherlands \\ ${ }^{4}$ Instituut voor Theoretische Fysica, Universiteit van Amsterdam, P. O. Box 94485, 1090 GL Amsterdam, The Netherlands
}

(Received 25 February 2011; published 30 June 2011)

\begin{abstract}
We investigate a hard-square lattice gas on the square lattice by means of transfer-matrix and Monte Carlo methods. The size of the hard squares is equal to two lattice constants, so the simultaneous occupation of nearest-neighbor sites as well as of next-to-nearest-neighbor sites is excluded. Near saturation of the particle density, this system is known to undergo a phase transition to one out of four partially ordered phases. We find that this transition displays strong finite-size corrections to scaling and that the correlation functions deviate from isotropy to rather large distances. In contrast with an earlier study, we find that the critical temperature exponent of the transition is not Ising-like.
\end{abstract}

DOI: 10.1103/PhysRevE.83.061153

PACS number(s): 64.60.De, 05.10.Ln, 68.35.Rh

\section{INTRODUCTION}

Consider adsorption of adatoms on the (100) surface of a cubic crystal. In the presence of attractive forces, the adatoms will tend to occupy positions directly above the centers of the faces of the square lattice defining the surface layer. It is obvious that the phase behavior of such a system depends on parameters such as the chemical potential of the adatoms and their size or, more generally, on the potentials describing the interactions involving the adatoms. If the adatoms are large enough, nearest-neighbor and next-to-nearest-neighbor sites will be excluded for a pair of adatoms. If the size of the adatoms allows the simultaneous occupation of third-neighbor sites separated by two lattice units, the behavior of the system may be, at least approximately, described by a lattice gas model defined by the partition sum

$$
Z_{\mathrm{lg}}=\sum_{\left\{\sigma_{n}\right\}} \prod_{\langle i j\rangle}\left(1-\sigma_{i} \sigma_{j}\right) \prod_{[k l]}\left(1-\sigma_{k} \sigma_{l}\right) \prod_{m} \mu^{\sigma_{m}},
$$

where the lattice variables $\sigma_{i}$ are labeled by their lattice site number $i$ and can take the values 0 (not occupied) and 1 (occupied). The particle number is controlled by the chemical potential $\mu$. The first product is on all nearest-neighbor pairs, and the second one is on all next-to-nearest-neighbor pairs. Interactions between adatoms at distances of two or more lattice units are neglected. The excluded area about each lattice gas particle assumes the shape of a square whose linear size equals two lattice units. The model is thus a hard-square model. It should not be confused with other hard-square models, such as Fisher's [1] and Baxter's [2,3], and the nearest-neighbor exclusion hard-square model without further interactions [4-9]. The latter model, as well as that of Eq. (1), are part of a family of hard-core lattice gases that are, apart from the size of the hard core, parametrized by the chemical potential as the only variable parameter. This family was recently investigated by means of Monte Carlo simulations in an extensive study by Marques Fernandes et al. [10] for hard-core sizes corresponding with exclusions up to the fifth neighbors. There are also other studies [11-15] that include the model of Eq. (1) as a specific case.

We proceed to summarize the existing understanding and results on the universality class of by Eq. (1). In the limit $\mu \rightarrow \infty$, the system will reach its densest state, which occupies one of four sites of the lattice of Eq. (1). That may be realized in a regular square pattern of the particles but one may then also independently shift horizontal (or vertical) rows of particles, without violating the neighbor exclusions of the system. The ground states are multiply degenerate, and the entropy density of the ground states of a system depends on the linear finite size $L$ as $1 / L$. For this reason, the model does not display long-range order in the sense of condensation of the lattice-gas particles on one out of four sublattices. But a different type of long-range order is still possible. Namely, in the limit $\mu \rightarrow \infty$, there will be either vertical or horizontal rows of particles, with every other site occupied. Moreover, these rows may be simultaneously shifted by one lattice unit in the direction perpendicular to the rows.

Thus, there are four distinct ordered phases. That is known to be a special and interesting case in the theory of phase transitions in two dimensions. As noted by Kinzel and Schick [11], such models typically belong to "nonuniversal" manifolds, so the symmetry properties of the model are not sufficient to predict its critical exponents, which suggests the use of numerical techniques. Since the Monte Carlo analysis of Marques Fernandes et al. [10] yielded estimates of the exponents that were close to the exact values of the Ising model, these authors described the transition as belonging to the Ising universality class.

The present work aims at a further investigation of this issue. The unavoidable error margins of the estimated exponents [10], which were not listed explicitly, leave some room for the possibility of critical behavior that differs from Ising universality. Furthermore, the available theoretical knowledge does not seem to provide evidence in favor of Ising universality. Examples of exactly solved models with four competing ground states are the Baxter model [16] and the AshkinTeller [17] model, which display a "nonuniversal" critical line with a temperature exponent that depends continuously on a parameter in the Hamiltonian of the model $[16,18]$, while their magnetic exponent remains constant at the Ising value $y_{t}=15 / 8$. For this reason, the magnetic exponent alone is not a good indicator of Ising universality.

Both the Baxter and the Ashkin-Teller model are composed of two interacting square-lattice Ising models. Here we shall 
show that this property also applies, at least in a broad sense, to the model of Eq. (1). First, we replace the labeling of the lattice sites as $i, j, k, \ldots$ by integer Cartesian coordinates $x, y$ running from 0 to $L-1$. We then define spin-1 Ising variables $s_{x, y}^{x}, s_{x, y}^{y}$ with possible values $0, \pm 1$ by

$$
\begin{aligned}
& s_{x, y}^{x}=\sigma_{x+1, y+1}+\sigma_{x+1, y}-\sigma_{x, y+1}-\sigma_{x, y} \\
& s_{x, y}^{y}=\sigma_{x+1, y+1}+\sigma_{x, y+1}-\sigma_{x+1, y}-\sigma_{x, y},
\end{aligned}
$$

for only even values of $x$ and $y$ in the range from 0 to $L-2$. Spin values +1 show that there is a particle in the right or upper side of an elementary square, and -1 for a particle on the opposite side. A spin value 0 denotes the absence of a particle. Note that the restriction that an elementary square contains 0 or 1 particles translates into $\left|s_{x, y}^{x}\right|=\left|s_{x, y}^{y}\right|$. The partition sum of this Ising-like model is

$$
\begin{aligned}
& Z_{\text {Ising }}=\sum_{\left\{s_{x, y}^{x}\right\}} \sum_{\left\{s_{x, y}^{y}\right\}} \prod_{x, y}\left\{\mu^{\left(s_{x, y}^{x} s_{x, y}^{y}\right)^{2}}\left[1-\left(s_{x, y}^{x}\right)^{2}-\left(s_{x, y}^{y}\right)^{2}+2\left(s_{x, y}^{x} s_{x, y}^{y}\right)^{2}\right]\right. \\
& \quad\left[1+\frac{1}{4} s_{x, y}^{x} s_{x+2, y}^{x}\left(1+s_{x, y}^{x}\right)\left(1-s_{x+2, y}^{x}\right)\right]\left[1+\frac{1}{4} s_{x, y}^{y} s_{x, y+2}^{y}\left(1+s_{x, y}^{y}\right)\left(1-s_{x, y+2}^{y}\right)\right] \\
& \quad\left[1-\frac{1}{16} s_{x, y}^{x} s_{x+2, y+2}^{x} s_{x, y}^{y} s_{x+2, y+2}^{y}\left(1+s_{x, y}^{x}\right)\left(1-s_{x+2, y+2}^{x}\right)\left(1+s_{x, y}^{y}\right)\left(1-s_{x+2, y+2}^{y}\right)\right] \\
& \left.\left[1-\frac{1}{16} s_{x, y}^{x} s_{x+2, y-2}^{x} s_{x, y}^{y} s_{x+2, y-2}^{y}\left(1+s_{x, y}^{x}\right)\left(1-s_{x+2, y-2}^{x}\right)\left(1-s_{x, y}^{y}\right)\left(1+s_{x+2, y-2}^{y}\right)\right]\right\} .
\end{aligned}
$$

The factors between square brackets are equal to 0 or 1 , thus imposing the various constraints. The first one guarantees that $\left|s_{x, y}^{x}\right|=\left|s_{x, y}^{y}\right|$; the second and the third ones represent neighbor exclusions in the $x$ and $y$ directions respectively; and the fourth and the fifth ones the remaining next-to-nearest-neighbor exclusion between particles in diagonally separated squares.

The model of Eq. (3) does not obey the spin-inversion symmetry of the usual Ising model. Instead, the model is invariant under the simultaneous inversion $s_{x, y}^{x} \leftrightarrow-s_{-x, y}^{x}$ of the $x$ spins and the spatial $x$ direction of the lattice. Whereas the separate factors in Eq. (3) do not obey this symmetry, the product does. Independently, Eq. (3) is also invariant under the simultaneous inversion $s_{x, y}^{y} \leftrightarrow-s_{x,-y}^{y}$. These symmetries still allow Ising-like order parameters $\left\langle s_{x}\right\rangle$ and $\left\langle s_{y}\right\rangle$, and in this sense the model of Eq. (3) is still Ising-like.

The first and last two factors between the square brackets in Eq. (3) specify a coupling between the $s^{x}$ and $s^{y}$ spins. In their absence, the model would decompose in two systems of linear chains in the $x$ and $y$ directions, without phase transitions. Their presence not only introduces a coupling between the chains in both directions but also can be associated with a coupling between the two Ising-like order parameters, similarly to the four-spin couplings in the Baxter model [16] and the Ashkin-Teller [17] model. In the latter two models, a positive coupling between the Ising-like order parameters corresponds to a value of the temperature exponent exceeding the Ising value $y_{t}=1$.

It is, thus, of interest to consider the signs of the four-spin products of the form $s_{x, y}^{x} s_{x+a, y+b}^{x} s_{x, y}^{y} s_{x+c, y+d}^{y}$ in Eq. (3). The first factor between square brackets contains a positive contribution, whereas the last two factors contain negative contributions. Since the latter ones are subject to four extra conditions expressed by factors such as $\frac{1}{2}\left(1+s_{x, y}^{x}\right)$, one is tempted to expect that the positive contribution dominates, which suggests that $y_{t}>1$, differing from Ising universality.
To resolve this issue, we performed transfer-matrix and Monte Carlo calculations. The numerical techniques are explained in Sec. II and the results are presented in Sec. III. While a rather large range of finite sizes has become available with the present transfer-matrix techniques and computers, our early optimism was not justified. The transfer-matrix analysis yielded puzzling results, including rather extreme deviations from isotropy, to the extent that one might doubt the asymptotic conformal invariance of the model of Eq. (3). This situation is quite unlike, e.g., the nearest-neighbor exclusion model, which allows [9] the very accurate determination of some universal parameters. It thus seemed necessary to apply Monte Carlo calculations, which can handle much larger system sizes, to analyze the model of Eq. (3). Our first round of Monte Carlo calculations using the METROPOLIS method [19], which covered a range of finite-sizes up to $L \approx 120$, did not yield a clear numerical picture either. For this reason we developed a more efficient Monte Carlo method, described in Sec. II B. With the help of this method, which moves a string of particles in addition to METROPOLIS moves, meaningful numerical results could be obtained for larger systems. These provide a satisfactory determination of the temperature exponent, as well as an explanation of the unusual behavior found in Sec. III A. Our results are briefly discussed in Sec. IV.

\section{NUMERICAL TECHNIQUES}

\section{A. The transfer matrix}

The calculation of a few leading eigenvalues of the transfer matrix yields the free energy density and the correlation lengths associated with correlation functions corresponding to subleading eigenvalues. Here we do not attempt to calculate the transfer matrix itself, but we build an algorithm that executes the multiplication of the transfer matrix with a given vector. 
The transfer matrix is decomposed in a number of sparse matrices that can be stored in a memory with a size roughly of the same order as the linear dimension of the transfer matrix. The principle and some details of the sparse-matrix decomposition are explained, e.g., in Refs. [8,20-23].

We apply the transfer-matrix method to systems that are periodic with the finite size $L$ in one direction, and consider the limit of infinite size for the other direction. Thus the model is wrapped on a cylinder. For a transfer matrix that adds one layer of $L$ sites to the system, the free energy density follows as

$$
f(L)=\frac{\zeta}{L} \log \lambda_{0},
$$

where $\lambda_{0}$ is the largest eigenvalue of the transfer matrix $\mathbf{T}$ and $\zeta$ is the geometrical factor defined as the ratio of length unit (circumference of the cylinder divided by $L$ ) over the thickness of a layer added by the action of $\mathbf{T}$. The correlation length $\xi_{k}(L)$ associated with the subleading eigenvalue $\lambda_{k}$ of $\mathbf{T}$ is given by

$$
\xi_{k}^{-1}(L)=\zeta \log \frac{\lambda_{0}}{\left|\lambda_{k}\right|} .
$$

For conformally invariant models the scaled correlation length satisfies [24] $X_{k}=L /\left[2 \pi \xi_{k}(L)\right]$, where $X_{k}$ is the scaling dimension of the observable whose decay of correlations is described by the characteristic length $\xi_{k}(L)$. Normally one expects nonzero irrelevant fields, so this equation applies only in the limit $L \rightarrow \infty$. In the presence of an irrelevant field, and of a small deviation of the chemical potential $\mu$ with respect to its critical value $\mu_{\mathrm{c}}$, scaling predicts that the scaled gap, defined as

$$
X_{k}(L, \mu) \equiv \frac{L}{2 \pi \xi_{k}(L, \mu)}
$$

behaves as

$$
X_{k}(L, \mu)=X_{k}+a_{k}\left(\mu-\mu_{\mathrm{c}}\right) L^{y_{t}}+b_{k} L^{y_{i}}+\ldots,
$$

where the temperature exponent $y_{t}$ is positive and the irrelevant exponent $y_{i}$ is negative.

The elements of the transfer matrix are non-negative, so the Perron-Frobenius theorem implies that elements of the eigenvector corresponding to the largest eigenvalue can be chosen non-negative. Iterative methods starting from a vector with only positive elements will thus yield the latter eigenvector and its eigenvalue. The determination of the subleading eigenvalues is facilitated by the use of their symmetry properties. Translations of the lattice correspond with rotations about the axis of the cylinder. The model is thus invariant under a discrete set of translations in the finite direction, and [in the lattice gas representation of Eq. (1)] also under an inversion of the coordinate in that direction. Each of these symmetry operations is represented by a matrix, say $\mathbf{S}$, that acts in the same vector space as the transfer matrix $\mathbf{T}$. Since $\mathbf{T}$ and $\mathbf{S}$ commute, there exists a common set of eigenstates of $\mathbf{T}$ and $\mathbf{S}$. The symmetry properties of the leading eigenvectors are found by inspection of numerically solutions of the eigenproblem for small systems. For larger systems we may then impose the desired symmetry on the vector that is being iterated. This will normally yield the eigenvector associated with the largest eigenvalue (or the one with the largest absolute value) in the subspace corresponding with that symmetry.

First, we consider the lattice model of Eq. (1) wrapped such that one set of edges of the square lattice is parallel to the axis of the cylinder. The transfer matrix algorithms for this model obviously includes the coding of the possible configurations of lattice gas particles on a circular row of $L$ sites at the end of the cylinder by means of subsequent integers. It thus seems natural to employ a coding algorithm that takes into account nearestas well as next-to-nearest-neighbor exclusion. The next-tonearest-neighbor exclusion, which applies to sites on different rows, does not impose further restrictions on the configuration of an $L$-site circular row, besides the restrictions imposed by the nearest-neighbor exclusion. The applicable type of coding was already employed and described in the literature [8,9]. However, the application of sparse-matrix techniques also requires the coding of particle configurations as obtained after adding a part of the next circular row of sites on top of an $L$-site circular row. This leads to a closed array consisting of $L+2$ sites with two "steps." The different geometry of the $L+2$-site array imposes additional restrictions at the "steps." These were included in the algorithm by simply putting the corresponding weights to zero. The resulting transfer matrix $\mathbf{T}_{\mathrm{a}}$ (where "a" denotes axial) is symmetric, and its largest eigenvalues were determined by maximization of the Rayleigh quotient using the conjugate-gradient method [25].

We also performed transfer-matrix calculations with a set of diagonals of the square lattice parallel to the axis of the cylinder. In this case the coding applies to a particle configuration on an $L$-site zigzag line. Nearest- as well as next-to-nearest-neighbor exclusion applies to the sites on this line. As before, the transfer matrix is decomposed in a number of sparse matrices. Application of these does not lead to a change in the number of sites at the end of the cylinder but introduces straight segments consisting of two lattice edges into the zigzag pattern. Along these straight parts, the next-to-nearest-neighbor exclusion does not apply. For this reason we employed the same coding as before and, where necessary, we enforced the next-to-nearest-neighbor exclusion by putting the applicable weights to zero. The resulting transfer matrix $\mathbf{T}_{\mathrm{d}}$ (where "d" denotes diagonal) is not symmetric, and the Hessenberg algorithm as described in Ref. [21] was employed to find its largest eigenvalues.

\section{B. Monte Carlo algorithm}

As already known from the literature, the phase transition of the model of Eq. (1) takes place at a particle density that is close to the value $1 / 4$ corresponding with full packing. This has unfortunate consequences for the efficiency of the METROPOLIS Monte Carlo method. There is only a small fraction of the lattice site that can accommodate another particle. When the algorithm visits an occupied site, the large value of the chemical potential renders it unlikely that the site will be vacated. Indeed, preliminary simulations of this type led to long autocorrelation times. Furthermore, this situation becomes rapidly worse with increasing system size, due to critical slowing down. 
We therefore explored ways to speed up the simulations. First, one may formulate a rejection-free algorithm by constructing the probability distribution of the lifetime of the initial state and use a random number to choose the lifetime. Then the type and location of the move can be chosen in accordance with the corresponding probabilities, with the help of two more random numbers.

We did not follow this path because we expected that nonlocal updates might be more effective. An existing algorithm is available called the geometric cluster algorithm [26]. It moves part of the particle configuration to a different location on the same lattice. The idea of such moves was introduced by Dress and Krauth [27] for hard particles in continuous space. We found that, although the geometric cluster algorithm is extremely efficient in simulations of lattice gases with nearest-neighbor exclusion [28], it is of less use when applied to the present model. It is helpful in realizing rapid equilibration between the different sublattices, but it does not reduce autocorrelation times associated with other properties. This is due to the tendency of the algorithm, when applied to dense systems, to form geometric clusters that include almost all of the lattice gas particles.

Another nonlocal method is suggested by the degrees of freedom that still exist in the limit $\mu \rightarrow \infty$, namely the freedom to shift a whole row of particles by one lattice unit in the direction of the row. At finite values of $\mu$, additional vacant sites will occur, and it may thus be possible to apply moves involving a part of a row. An update attempt consists of the following steps:

(i) Randomly choose a particle, and randomly choose a direction from the four possibilities.

(ii) Propose a move of the particle in the chosen direction. If this leads to a violation of the nearest-neighbor exclusion in that direction, shift that neighbor by one lattice unit in the same direction. If this leads to another nearest-neighbor conflict, apply another shift operation. Include as many neighbors in the chosen direction as necessary to avoid nearest-neighbor conflicts. This leads to the formation of a row of $L$ or less particles. The latter case arises if there are two neighboring vacancies on the line of the row.

(iii) Check if the proposed move leads to a violation of the next-to-nearest-neighbor exclusion at the end of the row. Accept the move if and only if there is no such violation.

Note that the aforementioned nearest-neighbor conflicts can arise only along the line of particles being constructed, because a nearest-neighbor conflict in the perpendicular direction would imply a next-nearest-neighbor violation in the initial configuration. Furthermore, next-to-nearest-neighbor conflicts can occur only at the end of the row. Other occurrences would also imply the existence of a conflict in the initial configuration.

It is easily verified that the reverse move has equal probability, so the algorithm satisfies the condition of detailed balance. It appears to have a reasonable acceptance ratio. It speeds up the calculation by a factor of about 100 in comparison with the METROPOLIS method. Since it conserves the particle number, and we use the chemical potential $\mu$ as the independent parameter, we applied it still in combination with METROPOLIS sweeps. This way of moving a row of particles is close in spirit to the "event chain" Monte Carlo moves described by Bernard et al. [29] for hard particles in continuous space. Typically one simulation cycle consisted of one METROPOLIS sweep, one geometric cluster step and 10 "sweeps" of attempts to move a row of particles. A sweep means here that $L^{2} / 4$ elementary squares were randomly chosen and, if it contained a particle, the update attempt started with that particle.

The correctness of the algorithm was confirmed by a number of tests comparing the results of METROPOLIS simulations with those of simulations including geometric cluster steps and/or attempts to move rows of particles. Further sensitive tests were performed by comparing the particle densities obtained by the Monte Carlo method with those from the transfer-matrix calculations. For chemical potentials $\mu<\mu_{\mathrm{c}}$, the various numerical results allow accurate extrapolations to the thermodynamic limit. The differences were as expected on the basis of the statistical accuracy of the Monte Carlo results.

\section{RESULTS}

\section{A. Transfer matrix results}

\section{Straight transfer}

On the basis of Eq. (7) one expects, at least for sufficiently large $L$, that a plot of the $X_{k}(L, \mu)$-versus- $\mu$ curves for different values of $L$ will display intersections converging to the point with coordinates $\mu_{\mathrm{c}}$ and $X_{k}$. Such a plot is shown in Fig. 1(a) based on the second largest (in absolute value) eigenvalue $\lambda_{1}$ of the transfer matrix $\mathbf{T}_{\mathrm{a}}$. This eigenvalue is a negative singlet, and the corresponding eigenvector is invariant under the symmetries mentioned in Sec. II A. Figure 1(a) shows finite-size data for the $X_{1}(L, \mu)$ in the range $6 \leqslant L \leqslant 36$. There are intersections, ranging from about $\mu=$ 4.4 for the smallest systems to about $\mu>5.7$ for the largest ones, without clear signs of convergence with increasing $L$. The estimates of the associated scaling dimension $X_{1}$ at these intersections decrease rapidly from about 0.02 to about 0.0015 . These values seem to extrapolate to $X_{1}=0$, in conflict with the expected value $1 / 8$. Furthermore, the corresponding values of $\mu$ do not seem to converge to the literature value quoted as $\mu_{\mathrm{c}}=4.574$ and $\mu_{\mathrm{c}}=4.578$ in Ref. [10].

Next we calculated the second subleading eigenvalue $\lambda_{2}$, i.e., the third largest one in absolute value. This eigenvalue is positive and the eigenvector is again invariant under the symmetries mentioned above. It was obtained using orthogonalization with respect to the leading eigenvector. The scaled gaps $X_{2}(L, \mu)$ are shown as a function of $\mu$ in Fig. 1(b). In this case there are also intersections. The chemical potential at the intersections increases with $L$ to about $\mu=4.66$ at the largest system size. These values are much closer to the existing result $\mu_{\mathrm{c}} \approx 4.576$ for the critical point [10]. However, the data do not allow a satisfactory extrapolation with $L$ and suggest that an extremum will occur for $L>36$. The behavior in this figure differs remarkably from that in Fig. 1(a).

The third subleading eigenvalue is a negative doublet, and the associated eigenstate is antisymmetric under a translation over $L / 2$ lattice units. The corresponding scaled gap $X_{3}(L, \mu)$ 

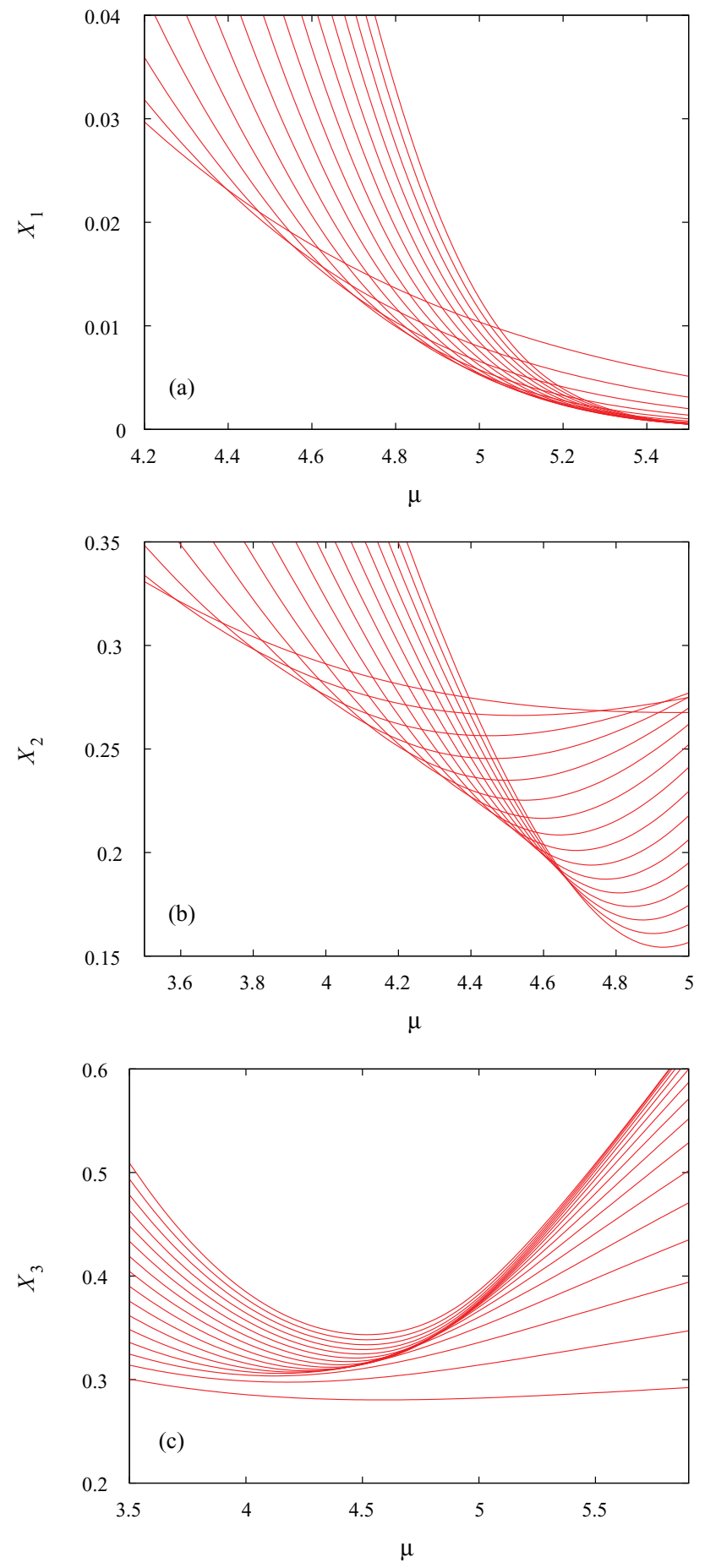

FIG. 1. (Color online) Scaled gaps $X_{1}(L, \mu), X_{2}(L, \mu)$, and $X_{3}(L, \mu)$ versus chemical potential $\mu$ for even system sizes $6 \leqslant L \leqslant$ 36. These data apply to the transfer matrix $\mathbf{T}_{\mathrm{a}}$ for transfer in the axial direction. For clarity, the data points, which lie at multiples of 0.1 on the $\mu$ scale, are connected by a smooth curve for each value of $L$. To avoid crowding, the data points are not shown individually. The scaled gaps increase with $L$ on the left-hand side.

is shown versus $\mu$ in Fig. 1(c). Intersections are absent for the smallest and the largest system sizes in the range $6 \leqslant L \leqslant 36$. These data suggest that we are still far away from the large- $L$ scaling limit.

\section{Diagonal transfer}

While a transfer matrix can be constructed that adds only $L / 2$ sites, we did not use it because it does not commute with the inversion operation. Instead, we used a transfer matrix adding $L$ sites, using a sparse-matrix decomposition that first sequentially adds $L / 2$ sites on the even positions, and then the same on the odd positions. Due to the orientation of the lattice edges, the unit of length is a factor $\sqrt{2}$ smaller than for the transfer matrix for transfer in the axial direction. The geometric factor is thus $\zeta=1 / 2$ in this case.

We performed calculations only for $L$ equal to multiples of 4 in order to avoid frustration effects due to the next-to-nearestneighbor exclusion. The leading eigenstate is again the fully symmetric Perron-Frobenius vector. The second eigenstate, as determined by calculations for small $L$, is a doublet with a negative eigenvalue. These two eigenvectors change sign under a translation of $L \sqrt{2}$ perpendicular to the transfer direction. They can be chosen such that one of them is symmetric under inversion and the other antisymmetric. Imposing the antisymmetry under translation and symmetry under inversion, we determined the eigenvalue $\lambda_{1}$ for larger systems up to $L=36$. The corresponding scaled gaps $X_{1}(L, \mu)$ are shown versus $\mu$ in Fig. 2(a). With exclusion of the smallest system size $L=4$, the intersections of the curves for sizes $L$ and $L+4$ lie in the interval $4.72<\mu<4.84$.

The numerical solutions for $\mu$ in the scaling equation

$$
X_{k}(L, \mu)=X_{k}(L+4, \mu)
$$

are denoted $\mu_{L}$. According to Eq. (7) they behave as

$$
\mu_{L}=\mu_{\mathrm{c}}+a L^{y_{i}-y_{t}}+\cdots .
$$

The data for $\mu_{L}$ are, however, not well described by a single correction term in $L$. Attempts to fit an expression with two powers of $L$ suggest that the critical point satisfies $\mu_{\mathrm{c}} \approx 4.6$ with an uncertainty of a few times $10^{-2}$.

We then calculated the eigenvalue $\lambda_{2}$ with the third largest absolute value. It is a positive singlet. Its eigenstate is translationally invariant, and it is antisymmetric under inversion. The results for the scaled gaps $X_{2}$ are shown in Fig. 2(b). Similar to the case of $X_{1}$, there are intersections near the existing result $\mu_{\mathrm{c}} \approx 4.576$ for the critical point, but the data are not well described by Eq. (9) and do not allow a convincing determination of $\mu_{\mathrm{c}}$.

The fourth largest eigenvalue $\lambda_{3}$ is positive singlet, and its eigenstate is fully symmetric. The corresponding scaled gaps are shown in Fig. 2(c) for system sizes in the range $8 \leqslant$ $L \leqslant 36$. Intersections are absent, and the data do not suggest convergence near the expected critical point.

Furthermore, we attempted to determine the conformal anomaly $c$ from the free energies [30,31] as calculated at $\mu=$ 4.585. Poor convergence prevented an accurate determination. Iterated fits with a fixed exponent -2 to the free energies calculated with the "axial" transfer matrix yielded results decreasing to $c=1.74$ for the largest $L$ available. The diagonal transfer matrix yielded estimates increasing to $c=0.68$ for the largest $L$.

Although the transfer-matrix method itself yields highly accurate eigenvalues and scaled gaps (typical errors are $10^{-12}$ or less), the present model apparently displays unusually 

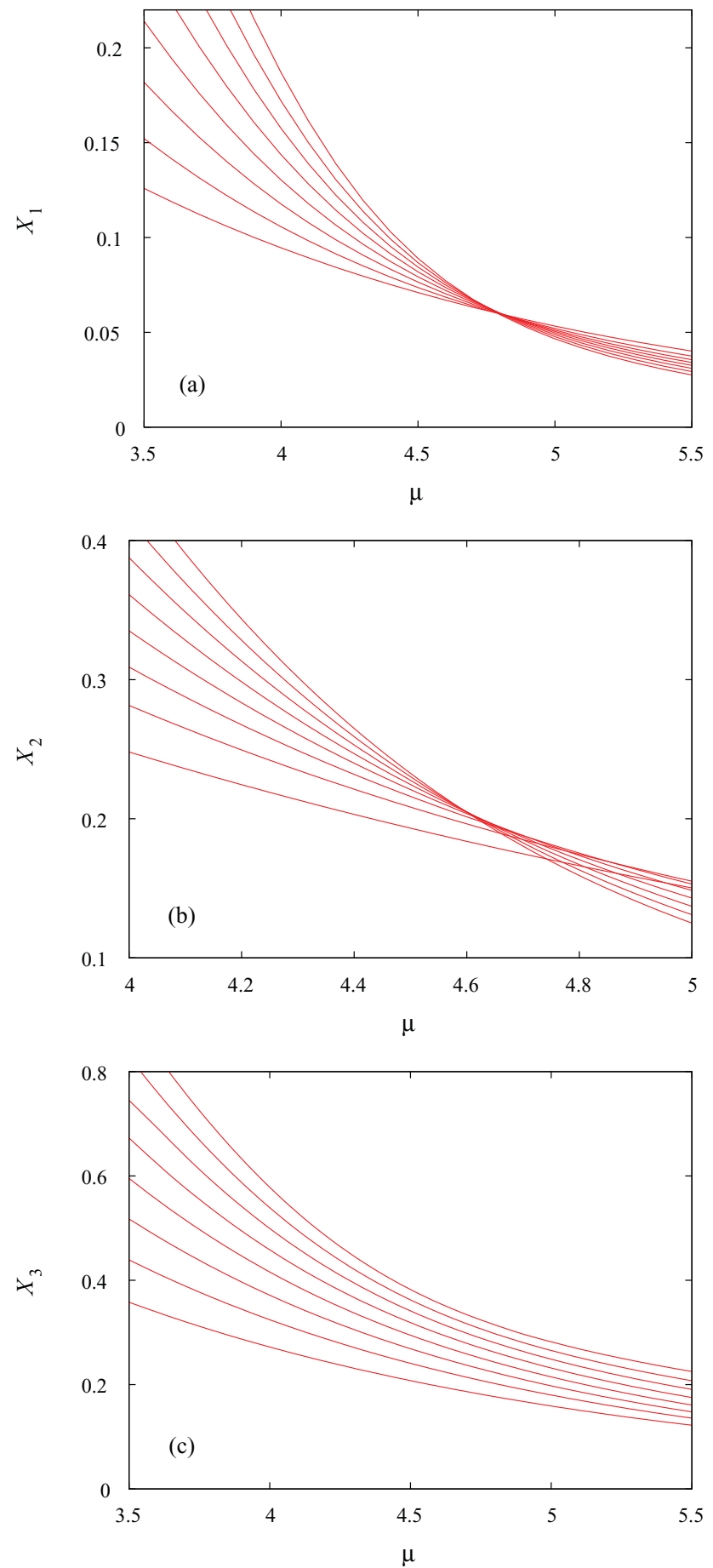

FIG. 2. (Color online) Scaled gaps $X_{1}(L, \mu), X_{2}(L, \mu)$, and $X_{3}(L, \mu)$ versus chemical potential $\mu$ for system sizes equal to multiples of 4 in the range $8 \leqslant L \leqslant 36$. These data apply to the transfer matrix $\mathbf{T}_{\mathrm{d}}$ for transfer in the diagonal direction. For clarity, the data points, which lie at multiples of 0.1 on the $\mu$ scale, are connected by a smooth curve for each value of $L$. To avoid crowding, the data points are not shown individually. The scaled gaps increase with $L$ on the left-hand side.

poor finite-size convergence, which thus prevents an accurate determination of the critical point and the universal quantities.

\section{B. Monte Carlo results}

The simulations were performed for systems on square lattices with periodic boundary conditions and 24 different linear sizes in the range $8 \leqslant L \leqslant 256$. The chemical potential was chosen close to the expected critical point $\mu \approx 4.58$, except for some additional runs to determine the dependence of the data on $\mu$. The length of the simulations per system size varied between at least $2 \times 10^{9}$ cycles for the systems with $L \leqslant 64$ to $4 \times 10^{8}$ cycles for $160 \leqslant L \leqslant 256$. Each cycle included, in addition to the Monte Carlo algorithms described in Sec. II B, the sampling of several quantities. These included the particle densities $\rho_{i}$ on the four sublattices labeled by $i \equiv 1+(x \bmod 2)+2(y \bmod 2)$ and the third neighbor correlations

$$
\begin{aligned}
& \gamma_{x, i}=4 L^{-2} \sum_{x, y \mid i} \sigma_{x, y} \sigma_{x+2, y} \\
& \gamma_{y, i}=4 L^{-2} \sum_{x, y \mid i} \sigma_{x, y} \sigma_{x, y+2},
\end{aligned}
$$

where the sums are over the sites $x, y$ on sublattice $i$.

The fluctuations in the sublattice densities are expressed by a quantity $\mathrm{m}^{2}$, analogously to the magnetization of a clock or Potts model, by

$$
m^{2}=\frac{4}{3} \sum_{i=1}^{4}\left(\rho_{i}-\rho\right)^{2},
$$

where $\rho \equiv\left\langle\sum_{i=1}^{4} \rho_{i}\right\rangle / 4$ is the total particle density. On this basis we can define a generalized susceptibility as

$$
\chi=L^{2}\left\langle m^{2}\right\rangle
$$

and a quantity $Q_{1}$ related to the Binder cumulant as

$$
Q_{1}=\frac{\left\langle m^{2}\right\rangle^{2}}{\left\langle m^{4}\right\rangle} .
$$

A more direct way to describe the type of order as it occurs in the ordered phases uses the third-neighbor correlations

$$
\begin{aligned}
& g_{x, e}=\gamma_{x, 1}+\gamma_{x, 2}, \\
& g_{x, o}=\gamma_{x, 3}+\gamma_{x, 4}, \\
& g_{y, e}=\gamma_{y, 1}+\gamma_{y, 3}, \\
& g_{y, o}=\gamma_{y, 2}+\gamma_{y, 4},
\end{aligned}
$$

from which we can form a generalized susceptibility

$$
\chi_{2}=L^{2}\left\langle\left(g_{x, e}-g_{x, o}\right)^{2}+\left(g_{y, e}-g_{y, o}\right)^{2}\right\rangle
$$

and a dimensionless ratio

$$
Q_{2}=\frac{\left\langle\left(g_{x, e}-g_{x, o}\right)^{2}+\left(g_{y, e}-g_{y, o}\right)^{2}\right\rangle^{2}}{\left\langle\left[\left(g_{x, e}-g_{x, o}\right)^{2}+\left(g_{y, e}-g_{y, o}\right)^{2}\right]^{2}\right\rangle} .
$$

\section{Universal ratios}

The finite-size scaling behavior of the dimensionless ratios $Q_{1}$ and $Q_{2}$ is obtained by writing these quantities in terms of derivatives of the free energy and application of a 
TABLE I. Parameters as determined by least-squares fits to the dimensionless ratios $Q_{1}$ and $Q_{2}$. Estimated error margins in the last decimal place are shown between parentheses.

\begin{tabular}{lcccc}
\hline \hline Parameter & \multicolumn{2}{c}{ Fit for $Q_{1}$} & \multicolumn{2}{c}{ Fit for $Q_{2}$} \\
\hline$Q$ & 0.890 & $(3)$ & 0.886 & $(3)$ \\
$\mu_{\mathrm{c}}$ & 4.585 & $(2)$ & 4.583 & $(2)$ \\
$y_{t}$ & 1.05 & $(2)$ & 1.05 & $(2)$ \\
$a_{1}$ & 0.0059 & $(5)$ & 0.0053 & $(5)$ \\
$a_{2}$ & -0.000069 & $(15)$ & -0.000066 & $(15)$ \\
$a_{3}$ & -0.0000013 & $(16)$ & -0.0000012 & $(16)$ \\
$b_{1}$ & 328 & $(58)$ & 323 & $(60)$ \\
$c$ & -154 & $(24)$ & -146 & $(24)$ \\
$L_{\text {min }}$ & 56 & - & 56 & - \\
\hline \hline
\end{tabular}

renormalization transformation with scale factor $L$. The result is given by

$Q(L, \mu)=Q+\sum_{k} a_{k}\left(\mu-\mu_{\mathrm{c}}\right)^{k} L^{k y_{t}}+c L^{2-2 y_{h}}+b L^{y_{i}}+\cdots$.

The term with exponent $2-2 y_{h}$ is the leading term due to the analytic part of the free energy, and $y_{i}$ is the leading irrelevant exponent. For Ising-like models, it is known that $y_{i}=-2$ but corrections with this exponent are also expected more generally.

As a first step, the difference $Q_{1}-Q_{2}$ was fitted by Eq. (17). These fits yielded a strong indication that the first term vanishes, i.e., that the universal constant $Q$ is the same for both types of data. Also the terms in the sum, and the one with exponent $y_{i}$ seem absent, in agreement with the assumption that $Q_{1}$ and $Q_{2}$ pertain to the same universal quantity, i.e., that the difference $Q_{1}-Q_{2}$ is due to the analytical background which behaves as $L^{2-2 y_{h}}$ in leading order.

The numerical data for $Q_{1}$ and $Q_{2}$ were also fitted separately by Eq. (17), using fixed values $y_{h}=15 / 8$ and $y_{i}=-2$, while the remaining parameters were left free. Many fits were made, with different values of the cutoff at small system sizes and also with additional corrections proportional to $L^{1-2 y_{h}}$ and $\left(\mu-\mu_{\mathrm{c}}\right) L^{2-2 y_{h}}$. Satisfactory fits were obtained only when restricting system sizes to $L \geqslant 56$ or 64 . Parameters as found on the basis of Eq. (17) are listed in Table I. We quote conservative error margins of four standard deviations to allow for the possible presence of additional correction terms in Eq. (17). This choice is consistent with the differences between the fits for $Q_{1}$ and $Q_{2}$, and with the change of the parameters due to variation of the fit formula and the cutoff at small system sizes. While the parameters $Q, \mu_{\mathrm{c}}$, and $y_{t}$, and to some extent also $a_{1}$, are rather stable under variations of the fits as mentioned above, the remaining parameters display larger differences.

\section{Susceptibilities}

The finite-size scaling behavior of the susceptibilities $Q_{1}$ and $Q_{2}$ is obtained from the scaling equation of the free energy
TABLE II. Parameters as determined by the least-squares fits to the susceptibilities $\chi_{1}$ and $\chi_{2}$. Estimated errors in the last decimal place are shown between parentheses. Fixed parameters are identified by the absence of error estimates.

\begin{tabular}{lcccc}
\hline \hline Parameter & \multicolumn{2}{c}{ Fit for $\chi_{1}$} & \multicolumn{2}{c}{ Fit for $\chi_{2}$} \\
\hline$y_{h}$ & 1.877 & $(5)$ & 1.877 & $(5)$ \\
$\chi_{0}$ & -54 & $(15)$ & -2280 & $(570)$ \\
$c_{0}$ & 0.0346 & $(20)$ & 1.33 & $(8)$ \\
$c_{1}$ & 0.00061 & $(3)$ & 0.0237 & $(13)$ \\
$c_{2}$ & 0.0000021 & $(6)$ & -0.00005 & $(2)$ \\
$c_{3}$ & 0.0000000 & $(1)$ & -0.000007 & $(8)$ \\
$b_{1}$ & 175 & $(56)$ & 7200 & $(2200)$ \\
$b_{2}$ & -700 & $(260)$ & -26700 & $(10000)$ \\
$\mu_{\mathrm{c}}$ & 4.584 & - & 4.584 & - \\
$y_{t}$ & 1.05 & - & 1.05 & - \\
$L_{\min }$ & 64 & - & 64 & - \\
\hline \hline
\end{tabular}

and taking the second derivative to the magnetic field. This leads to

$$
\begin{aligned}
\chi(L, \mu)= & \chi_{0}+\chi_{1}\left(\mu-\mu_{\mathrm{c}}\right)+L^{2 y_{h}-2}\left\{\sum_{k=0,1,2, \cdots} c_{k}\left(\mu-\mu_{\mathrm{c}}\right)^{k} L^{k y_{t}}\right. \\
& \left.+b_{1} L^{y_{1}}+\left[b_{2}+b_{21}\left(\mu-\mu_{\mathrm{c}}\right)\right] L^{y_{2}} \cdots\right\} .
\end{aligned}
$$

The numerical data for $\chi_{1}$ and $\chi_{2}$ were fitted by Eq. (18), using fixed values $\mu_{\mathrm{c}}=4.584, y_{t}=1.05, y_{1}=-2$, and $y_{2}=-2.75$, while the remaining parameters were left free. Reasonable fits were obtained only using system sizes $L \geqslant 64$. The $\mu$-dependent correction terms with amplitudes $\chi_{1}$ and $b_{21}$ were necessary to obtain reasonable residuals $\chi^{2}$ and consistency between both results for the universal parameter $y_{h}$ as obtained from the fits for $\chi_{1}$ and $\chi_{2}$. Some of the fitted parameters are summarized in Table II. One- $\sigma$ error estimates are listed. They do not include the uncertainties due to the error margins in the fixed parameters.

\section{Energy-like quantities}

The temperature exponent $y_{t}$, which was estimated in Sec. III B 1 from the dependence of the ratios $Q$ on the chemical potential, can also be determined from quantities involving the density $\rho$ at the critical point. For instance, the leading terms in the scaling behavior of the critical density are given by $\rho\left(L, \mu_{\mathrm{c}}\right)=\rho_{\mathrm{c}}+p L^{2-y_{t}}+\cdots$ with additional terms due to the irrelevant field and $\mu-\mu_{\mathrm{c}}$. A numerical analysis of the density suggests $y_{t}=1.05(5)$, which is consistent with, but less accurate than, the result in Sec. III B 1. We also made an attempt to determine $y_{t}$ by means of an analysis of the finite-size data for the specific-heat-like quantity $c \equiv L^{2}\left(\left\langle\rho^{2}\right\rangle-\langle\rho\rangle^{2}\right)$. This attempt was unsuccessful because its leading singular behavior, which goes as $L^{2 y_{t}-2}$, is not sufficiently different from the analytic background which is independent of $L$. Next, we considered the connected correlation $A(L, \mu)$ between the squared magnetization as defined in Sec. III B and the density

$$
A(L, \mu) \simeq \ll m^{2} \rho \gg=\left\langle m^{2} \rho\right\rangle-\left\langle m^{2}\right\rangle\langle\rho\rangle .
$$

Its finite-size scaling behavior near criticality follows by differentiation of the free energy, twice to a sublattice field 
and once to the chemical potential. The statistical analysis of the data in terms of this scaling expression thus yields an independent determination of the temperature exponent. It led to results for $y_{t}$ between 1.05 and 1.10, depending on the number of correction terms. Here, the error estimation for $y_{t}$ is affected by the lack of precise knowledge of the error in the finite-size data for the largest system sizes. This situation occurs when the autocorrelation time becomes of the same order as the run length. This problem is more serious for $A(L, \mu)$ than for other sampled quantities, because the random sampling of higher derivatives of the free energy appears to be more sensitive to large and infrequent fluctuations. We also investigated the related quantity

$$
B(L, \mu) \simeq\left(\ll m^{2} \rho \gg=\left\langle m^{2} \rho\right\rangle-\left\langle m^{2}\right\rangle\langle\rho\rangle\right) /\left\langle m^{2}\right\rangle,
$$

in which one may hope that some of the magnetization fluctuations will cancel. But the scatter of data points taken between runs at the same chemical potential still suggested that the errors within the runs are underestimated. Expression of $B(L, \mu)$ in derivatives of the free energy and expansion in $\mu-\mu_{\mathrm{c}}$ yields

$$
\begin{aligned}
B(L, \mu)= & L^{y_{t}-2}\left(\sum_{j=0,1,2, \cdots} c_{j} L^{j y_{t}}\left(\mu-\mu_{\mathrm{c}}\right)^{j}+b_{1} L^{y_{1}}\right. \\
& \left.+b_{2} L^{y_{2}}+\cdots\right)+L^{2-2 y_{h}}\left[a_{0}+a_{1}\left(\mu-\mu_{\mathrm{c}}\right)+\cdots\right] .
\end{aligned}
$$

Fits of this expression to the Monte Carlo data yielded estimates of $y_{t}$ clustered at 1.07 to 1.08 , with error margins of 0.01 to 0.02 , depending on the number of correction terms used in the fit formula and the cutoff at small system sizes. In view of the difficulties due to the error estimation, we consider this result for $y_{t}$ as somewhat tentative.

\section{Magnetic correlation functions}

The sampling of correlations over distances $r$ in a system of finite size $L$ will yield $L$-dependent correlation functions $g(L, r)$, but here we are interested in the limit of infinite system size $g(r) \equiv \lim _{L \rightarrow \infty} g(L, r)$. Scaling predicts that, at criticality,

$$
g(L, r)=r^{2 y_{h}-4} \tilde{g}(L / r),
$$

where corrections to scaling are neglected, so this formula applies only for sufficiently large $r$. The universal scaling function $\tilde{g}(L / r)$ rapidly approaches a constant for large $L / r$, which corresponds with a small finite-size dependence of $g(L, r)$ for $L \gg r$. Taken together with Eq. (22), this enables the estimation of $g(r)$ from simulation results for $g(L, r)$ with sufficiently large $L$. To facilitate this estimation, we restrict the finite sizes $L$ as well as the distances $r$ to powers of 2 . From Eq. (22) we find that

$$
g\left(L, 2^{p}\right) / g\left(2 L, 2^{p}\right)=\tilde{g}\left(2^{-p} L\right) / \tilde{g}\left(2^{1-p} L\right),
$$

where $p$ determines the distance as $r=2^{p}$. Thus, from simulation results for sizes $L$ and $2 L$ we numerically determine the ratios $\tilde{g}(4) / \tilde{g}(2), \tilde{g}(8) / \tilde{g}(4)$, and $\tilde{g}(16) / \tilde{g}(8)$. For $L / r \geqslant 16$

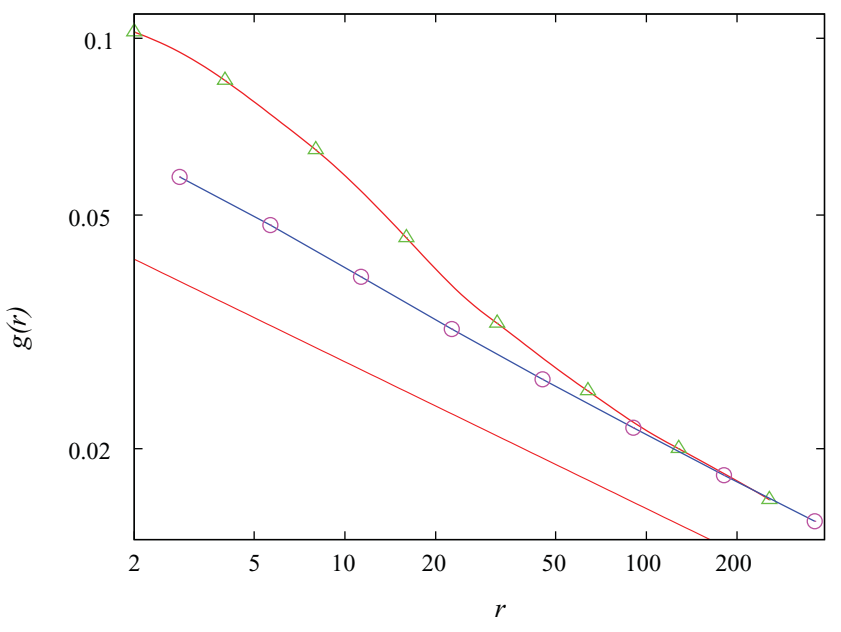

FIG. 3. (Color online) Correlation functions $g_{\mathrm{a}}(r)$ (triangles) and $g_{\mathrm{d}}(r)$ (circles) as a function of distance $r$. These functions describe sublattice-sublattice correlations in two different directions. The numerical errors are smaller than the symbol sizes, except for the largest values of $r$ where they are about the same. The curves are added for clarity. The straight line corresponds with decay as $r^{-1 / 4}$ and serves only for the purpose of comparison with the Monte Carlo data.

the correlation function $g(L, r)$ depends only weakly on $L$, and we neglect this dependence, i.e., we set $\tilde{g}(\infty)=\tilde{g}(16)$. Combining the numerical results for these ratios, one obtains the ratios $\tilde{g}(\infty) / \tilde{g}(L / r)$ for $L / r=2,4,8, \ldots$. These ratios serve as correction factors to estimate the infinite-system correlation function as

$$
g(r)=\frac{\tilde{g}(\infty)}{\tilde{g}\left(L_{\max } / r\right)} g\left(L_{\max }, r\right),
$$

where $L_{\max }=512$ is the largest system that was simulated. The correction factors $\tilde{g}(L / r) / \tilde{g}(L / 2 r)$ were determined from data for system sizes 512 and 256 and independently for sizes 256 and 128. On this basis we are confident that the procedure leads to results for correlation functions $g(r)$, as defined below, with errors not exceeding a few percent.

We define a sublattice correlation function $g_{\mathrm{a}}$ along the direction of the lattice axes and another one $g_{\mathrm{d}}$ in the diagonal direction:

$$
g_{\mathrm{a}}(r) \equiv\left\langle\sigma_{x, y} \sigma_{x+r, y}\right\rangle-\rho^{2}
$$

and

$$
g_{\mathrm{d}}(r \sqrt{2}) \equiv\left\langle\sigma_{x, y} \sigma_{x+r, y+r}\right\rangle-\rho^{2}
$$

These quantities were sampled during simulations of systems with sizes $L=128,256$, and 512 for $\mu=4.58$, which is close to the critical point. Data were taken for $r$ equal to powers of 2 up to $L / 2$. The results are shown in Fig. 3. These data show that the density-density correlation function on the sublattices is larger along the edge direction than along the diagonal direction. The difference persists until distances exceeding 100 lattice units. 


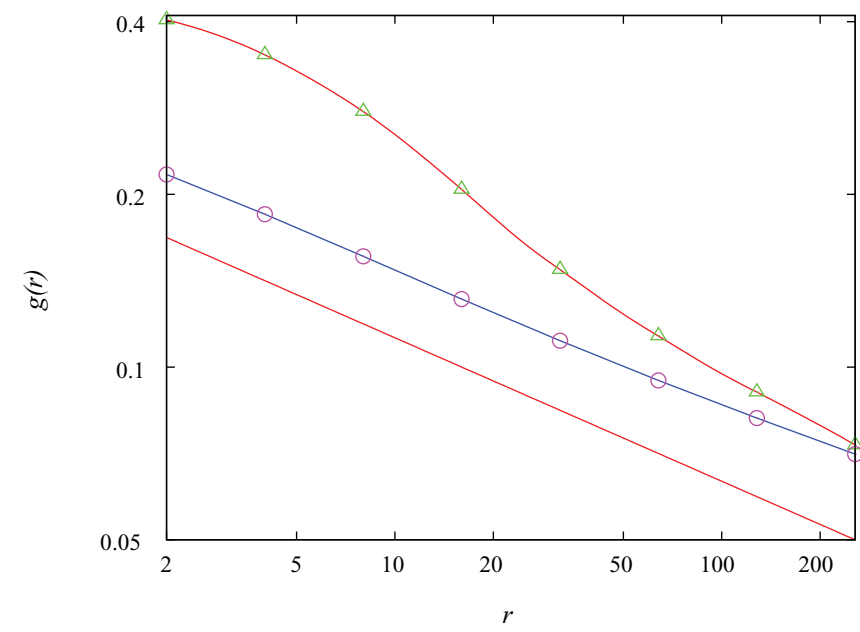

FIG. 4. (Color online) Correlation functions $g_{1}(r)$ and $g_{2}(r)$ as a function of distance $r$. These functions describe the correlations in the $x$ direction between the $x$ and $y$ components of the magnetization in the spin representation of the present lattice gas model. The numerical errors are smaller than the symbol sizes, except for the largest values of $r$ where they are about the same. The curves are added for clarity. The straight line corresponds with decay as $r^{-1 / 4}$ and serves only for the purpose of comparison with the Monte Carlo data.

Two more types of correlations were determined. They are conveniently described in terms of the three-valued spins $s^{x}$ and $s^{y}$ defined earlier in each elementary square:

$$
g_{1}(r) \equiv\left\langle s_{x, y}^{x} s_{x+r, y}^{x}\right\rangle
$$

and

$$
g_{2}(r) \equiv\left\langle s_{x, y}^{y} s_{x+r, y}^{y}\right\rangle .
$$

These correlation functions describe the decay of the correlations for each of the two spin components. The data for system sizes up to $L=512$ were corrected for the finite-size effect as described above. The results are shown in Fig. 4. It appears that the anisotropy of the pair $g_{1}, g_{2}$ is even more prominent than that of $g_{\mathrm{a}}, g_{\mathrm{d}}$. Nevertheless, the results shown in Fig. 4 suggest that the anisotropy disappears at distances exceeding a few hundred lattice units.

\section{DISCUSSION}

The behavior of the correlation lengths found by the transfer-matrix calculations in Sec. III A does not seem to agree with a simple scaling limit. Some of the scaled gaps yield intersections that lie in the neighborhood of the expected critical point but do not allow accurate extrapolations, and the value of the scaled gap does not seem to converge to the expected scaling dimensions. Some other scaled gaps do not even approximately agree with the expected behavior near a critical point. Moreover, the results for the smallest gap, which we interpret as describing the magnetic correlation function, display a pronounced difference between the axial and the diagonal directions. The dissimilarity between these directions is such that one might even doubt whether the present lattice-gas model satisfies asymptotic conformal invariance at criticality. This anisotropic behavior resembles that of an exactly solved tiling model $[32,33]$ of a plane by triangles and squares. The latter model satisfies scale invariance but not conformal invariance, and its asymptotic behavior is that of two decoupled, anisotropic free-fermion models [34]. Because the anisotropy of the two models has the opposite sign, it cannot be absorbed by an anisotropic scaling of the lattice. However, the analysis of correlations of the present lattice-gas model over a much longer range than what can be accessed by transfer-matrix methods indicates that the anisotropy vanishes at large distances and thus shows behavior consistent with asymptotic conformal invariance. This interpretation is also consistent with the expected value $c=1$ of the conformal anomaly in relation with the finite-size estimates quoted in Sec. III A 2.

The transfer-matrix results, especially those of the "edge" transfer matrix, should be interpreted in terms of the absence of finite-size convergence in the range of available system sizes. It is quite clear that the correlation functions do not reach their asymptotic regime within the range $L \leqslant 36$ of available finite sizes. The fact that the "edge" transfer matrix yields results that are even worse than the "diagonal" one can be related to the inequivalence of the $x$ and $y$ directions in the cylindrical geometry. This becomes already clear by inspection of the ground states, which consist of rows of particles, all of which are in either the $x$ or in the $y$ direction. Each such row can take two distinct configurations, related by a shift in the direction of the row. For a long cylinder, this twofold degeneracy leads to a strong tendency for the formation of such rows perpendicular to the axis of the cylinder, instead of parallel. This effect is purely entropic. It leads to longranged correlations in the length direction of the cylinder, associated with the occupation difference between the odd and the even rows. These correlations can thus be associated with the negative eigenvalue $\lambda_{1}$, in line with the small values of the scaled gaps near the critical point as shown in Fig. 1(a). For transfer in the diagonal direction, the $x$ and $y$ directions remain equivalent and the transfer-matrix results for $X_{1}$ and $X_{2}$ seem better behaved, although the values of the scaled gaps do not agree with the expected scaling dimensions. Our interpretation is that they are still subject to relatively strong corrections to scaling and that exposure of the true asymptotic behavior requires much larger system sizes.

This interpretation is supported by the analysis of the Monte Carlo results, which leads to consistent results only for system sizes exceeding 48 or 64 . The analysis of the correlation functions shows that asymptotic isotropy is reached only for system sizes of several hundred lattice units. Although our data analysis for systems larger than about $L=64$ leads to acceptable residuals, one has to keep in mind that the remaining range of system sizes is not wide; accordingly, we quoted conservative errors of four $\sigma$ in Table I. Also taking into account the independent determination of the temperature exponent in Sec. III B 3, we present our final estimate as $y_{t}=1.06(3)$.

Even with this conservative error estimate, the deviation of $y_{t}$ from the Ising universal value is significant. Together with the absence of a theoretical argument in favor of Ising-like behavior, it thus seems very likely that the universality class of the present lattice gas is not Ising-like and corresponds instead with a Baxter model or an Ashkin-Teller model with a positive four-spin coupling. This interpretation is consistent 
with our numerical result for $y_{h}$, which is close to the expected value 15/8. However, the statistical error listed in Table II has still to be revised to include the effect of the uncertainty in the critical point, which we quote as $\mu_{\mathrm{c}}=4.584(2)$ on the basis of the results in Table I. This leads to our final estimate $y_{h}=1.877(9)$.

\section{ACKNOWLEDGMENTS}

This research is supported by the NUAA Research Funding (People's Republic of China) under Grant No. NS2010155. One of us (H.B.) thanks the Lorentz Fund (The Netherlands) for support.
[1] M. E. Fisher, J. Math. Phys. 4, 278 (1963).

[2] R. J. Baxter, J. Phys. A 13, L61 (1980).

[3] D. A. Huse, J. Phys. A 16, 4357 (1983).

[4] D. S. Gaunt and M. E. Fisher, J. Chem. Phys. 43, 2840 (1965).

[5] D. W. Wood and M. Goldfinch, J. Phys. A 13, 2781 (1980).

[6] Z. Racz, Phys. Rev. B 21, 4012 (1980).

[7] G. Kamieniarz and H. W. J. Blöte, J. Phys. A 26, 6679 (1993).

[8] S. Todo and M. Suzuki, Int. J. Mod. Phys. C 7, 811 (1996).

[9] W. Guo and H. W. J. Blöte, Phys. Rev. E 66, 046140 (2002).

[10] H. C. Marques Fernandes, J. J. Arenzon, and Y. Levin, J. Chem. Phys. 126, 114508 (2007).

[11] W. Kinzel and M. Schick, Phys. Rev. B 24, 324 (1981).

[12] J. Amar, K. Kaski, and J. D. Gunton, Phys. Rev. B 29, 1462 (1984).

[13] K. Binder and D. P. Landau, Phys. Rev. B 21, 1941 (1980).

[14] N. G. Almarza, J. A. Capitán, J. A. Cuesta, and E. Lomba, J. Chem. Phys. 131, 124506 (2009).

[15] M. E. Zhitomirsky and H. Tsunetsugu, Phys. Rev. B 75, 224416 (2007).

[16] R. J. Baxter, Phys. Rev. Lett. 26, 832 (1971).

[17] J. Ashkin and E. Teller, Phys. Rev. 64, 178 (1943).

[18] L. P. Kadanoff, Phys. Rev. Lett. 39, 903 (1977); L. P. Kadanoff and A. C. Brown, Ann. Phys. 120, 39 (1979).

[19] N. Metropolis, A. Rosenbluth, M. Rosenbluth, A. Teller, and E. Teller, J. Chem. Phys. 21, 1087 (1953).
[20] H. W. J. Blöte and M. P. Nightingale, Physica A 112, 405 (1982).

[21] H. W. J. Blöte and B. Nienhuis, J. Phys. A 22, 1415 (1989).

[22] H. W. J. Blöte, F. Y. Wu, and X. N. Wu, Int. J. Mod. Phys. B 4, 619 (1990).

[23] H. W. J. Blöte and M. P. Nightingale, Phys. Rev. B 47, 15046 (1993).

[24] J. L. Cardy, J. Phys. A 17, L385 (1984).

[25] M. R. Hestenes and E. Stiefel, J. Res. Natl. Bur. Stand. 49, (1952); see also K. E. Atkinson, An Introduction to Numerical Analysis, 2nd ed. (John Wiley \& Sons, New York, 1989).

[26] J. R. Heringa and H. W. J. Blöte, Phys. Rev. E 57, 4976 (1998).

[27] C. Dress and W. Krauth, J. Phys. A 28, L597 (1995).

[28] J. R. Heringa and H. W. J. Blöte, Physica A 232, 369 (1996); 251, 224 (1998).

[29] E. P. Bernard, W. Krauth, and D. B. Wilson, Phys. Rev. E 80, 056704 (2009).

[30] H. W. J. Blöte, J. L. Cardy, and M. P. Nightingale, Phys. Rev. Lett. 56, 742 (1986).

[31] I. Affleck, Phys. Rev. Lett. 56, 746 (1986).

[32] M. Widom, Phys. Rev. Lett. 70, 2094 (1993).

[33] P. A. Kalugin, J. Phys. A 27, 3599 (1994).

[34] D. Dei Cont, Ph.D. thesis, University of Amsterdam (2005). Further information is available on request via one of the authors (B.N.). 